\title{
Interband Absorption and Luminescence in InP/InAs/InP Spherical Core/Shell/Shell Heterostructure for Moderate Regime of Size Quantization
}

\author{
V.A. Harutyunyan, E.M. Kazaryan and D.B. Hayrapetyan* \\ Russian-Armenian University, 123 Hovsep Emin Str., Yerevan 0051, Armenia
}

(Received January 29, 2020; in final form March 24, 2020)

\begin{abstract}
The states of charge carriers in the spherical InP/InAs/InP core/shell/shell heterostructure are considered. Consideration was carried out for the case of "moderate" quantization, when the energy of electrostatic interaction of an electron and hole is comparable to the energy of their size quantization in an InAs layer. The analytical form of the electron-hole interaction effective potential for the selected relations between the geometric sizes of the sample is determined. The wave functions and energy levels of charge carriers in the presence of this potential are calculated, too. It is shown that taking into account the electron-hole electrostatic interaction leads to an effective narrowing of the width of the band gap of the sample. The values of this effective narrowing is calculated for different values of the layer thickness. Interband optical absorption and photoluminescence in this heterostructure are also considered. With increase of the layer thickness, the absorption and luminescence peaks are shifted to the low frequency range of the absorbed or emitted light.
\end{abstract}

DOI: 10.12693/APhysPolA.137.1168

PACS/topics: InP/InAs/InP spherical core/shell/shell, electron-hole interaction, interband absorption, recombination energy, photoluminescence

\section{Introduction}

Along with many low-dimensional systems different semiconductor spherical core/shell/shell (quantum dotquantum well) heterostructures have been studied intensively both experimentally and theoretically during the last three decades (see [1-10] and references therein). In such layered structures the energy configuration of charge carriers is defined by the geometric sizes of the structure's components and by the relations between the corresponding physical parameters of contacting materials. Thus, one of the distinctive features of such core/shell/shell nanomaterials is the compositional dependence of their electronic, optical, kinetic and other properties. From this point of view, the study of spherical core/shell/shell structures with InP and InAs components is of undoubted interest. This is due to the reality that these semiconductor compounds are the most widely used in various devices of solid-state electronics. Moreover, they are used both separately [11-14] and jointly [15-25] as components in the composition of various composite heterostructures. The electron affinity $U_{c}$ and the band gap $E_{g}$ for InP and InAs have the following values: $U_{c}=-4.38 \mathrm{eV}, E_{g}=1.344 \mathrm{eV}$-for $\mathrm{InP}$, and $U_{c}=-4.9 \mathrm{eV}, E_{g}=0.354 \mathrm{eV}$ for InAs [26-35], respectively.

*corresponding author; e-mail: david.hayrapetyan@rau.am
In the spherical core/shell/shell of the $\operatorname{InP} / \operatorname{InAs} / \operatorname{InP}$ heterocomposite for these contacting materials the energy gap at the interface will be $\Delta U_{c}=0.52 \mathrm{eV}$ for electrons of the conduction band and $\Delta U_{v}=0.47 \mathrm{eV}$ for holes of the valence band, respectively. That is, the InAs layer in this composition will act as quantum well for the charge carriers. The Bohr radius of bulk exciton for these materials has the following values: $a_{\mathrm{ex}}=10-12 \mathrm{~nm}$ for InP, and $a_{\mathrm{ex}}=30-35 \mathrm{~nm}$ for InAs, respectively [26-35]. In the presence of the above characteristics of the $\mathrm{InP} / \mathrm{InAs} / \mathrm{InP}$ heterostructure's components the states of charge carriers will be determined already by the relations between the geometrical dimensions of the components of this structure.

In the previous work, the authors have considered single-particle states and optical transitions in the spherical core/shell/shell structure when the strong quantization regime for charge carriers takes place in the $\mathrm{InP} / \mathrm{InAs} / \mathrm{InP}$ structure in the strong quantization mode for charge carriers in the InAs layer. In this case the layer thickness $L$ is much smaller than the Bohr radius of the bulk exciton $a_{\mathrm{ex}}$ in the material of the layer. Accordingly, the electrostatic interaction between the electron and the hole can be neglected in comparison with the energy of their size quantization in the layer [35].

In the present work, single-particle states and optical transitions in the spherical core/shell/shell structure of the $\mathrm{InP} / \mathrm{InAs} / \mathrm{InP}$ heterocomposition are theoretically investigated when the electrostatic interaction energy between an electron and a hole in InAs layer becomes comparable to their size quantization energy in the layer. 


\section{Single-particle states} in core $/$ shell/shell structure

Before proceeding to the theoretical calculations let us bring material parameters of InP and InAs crystals. Table I shows the necessary further characteristics of ordinary InP and InAs bulk crystals.

The sizes of the InP core and the outer InP shell will be considered such (of the order of 25-35 nm) that an electron and a hole can bind into a 3D exciton and the quantum size effect is manifested already for $3 \mathrm{D}$ excitons, but not for charge carriers. Let us turn now to the consideration of charge carriers' states in the InAs layer. As it is well known, the quantum size effect, as well as the exciton states, are most pronounced in the lower energy states. Accordingly, in further calculations, we will consider only first two energy states of charge carriers in the InAs layer. As already noted, we will consider such interval of values of the InAs layer's thickness, when the energy of the Coulomb interaction between the electron and the hole can no longer be neglected. For a radius of $3 \mathrm{D}$ exciton in $\mathrm{InAs}, \approx 35 \mathrm{~nm}$, we will consider the layer thickness in the range $L=R_{2}-R_{1}=20-35 \mathrm{~nm}$. Here, $R_{1}, R_{2}$ are the inner and outer radii of the layer, respectively. Therefore, the size-quantization energy of carriers $E_{\text {conf }}(L, \mu) \approx \frac{\hbar^{2}}{2 \mu L^{2}}$ will vary within the range (i) from $E_{\text {conf }}^{c}\left(L=20 \mathrm{~nm}, \mu_{c}\right) \approx 4.1344 \mathrm{meV}$ to $E_{\text {conf }}^{c}\left(L=35 \mathrm{~nm}, \mu_{c}\right) \approx 1.3501 \mathrm{meV}$ for electrons from the $c$-band (where $E_{\text {conf }}^{c} \ll \Delta U_{c}=520 \mathrm{meV}$ ), and (ii) from $E_{\text {conf }}^{v}\left(L=20 \mathrm{~nm}, \mu_{h}\right) \approx 0.2319 \mathrm{meV}$ to $E_{\text {conf }}^{v}\left(L=35 \mathrm{~nm}, \mu_{h}\right) \approx 0.0757 \mathrm{meV}$ for holes from $v$-band (where $E_{\text {conf }}^{v} \ll \Delta U_{v}=470 \mathrm{meV}$. Here, $\mu_{c}, \mu_{h}$ are the effective masses of electron and hole, respectively.

As one can see, for lower states the InAs layer in the radial direction can be approximated with high accuracy by the infinitely deep potential well. At the same time, this means that for these states the carrier infiltration from the InAs layer into the core and the outer InP shell will be practically absent and in the lower energy states the localization of carriers will only take place exactly in the InAs layer.

The wave functions of single-particle states are represented in the standard form

$$
\begin{aligned}
& \Psi_{n, l, m}(r, \vartheta, \varphi)=\Phi_{n, l}(r) Y_{l, m}(\vartheta, \varphi), \quad n=1,2,3, \ldots, \\
& l=0,1,2, \ldots, \quad m=0, \pm 1, \pm 2, \cdots \pm l .
\end{aligned}
$$

In the future, we will consider only states, when $l=0$. Taking into account the Coulomb interaction between the electron and the hole in this case one can write for the Schrödinger equation of the interacting electron-hole pair

$$
\begin{aligned}
& -\frac{\hbar^{2}}{2 \mu_{e}} \Delta_{c} \Phi\left(r_{c}, r_{v}\right)-\frac{\hbar^{2}}{2 \mu_{h}} \Delta_{v} \Phi\left(r_{c}, r_{v}\right) \\
& +U\left(r_{c}, r_{v}\right) \Phi\left(r_{c}, r_{v}\right)=E^{c, v} \Phi\left(r_{c}, r_{v}\right) .
\end{aligned}
$$

Here $\Delta_{c}, \Delta_{v}$ are the radial parts of the Laplace operator, $\Phi\left(r_{c}, r_{v}\right)$ and $E^{c, v}$ are the envelope wave functions and the energies of the pair, respectively.

\section{TABLE I}

Characteristics of InP and InAs bulk semiconductors. Data is taken from [26-35]. Note: $m_{0}$ is the free electron mass.

\begin{tabular}{l|c|c}
\hline \hline \multicolumn{1}{c|}{ Material } & InP & InAs \\
\hline Band gap $E_{g}[\mathrm{meV}]$ & 1344 & 354 \\
Electron eff. mass $\mu_{e} / m_{0}$ & 0.08 & 0.023 \\
Hole eff. mass $\mu_{h} / m_{0}$ & 0.6 & 0.41 \\
Electron affinity $U_{c}[\mathrm{meV}]$ & -4380 & -4900 \\
3D exciton radius $a_{\mathrm{ex}}[\mathrm{nm}]$ & $10-12$ & $30-35$ \\
3D exciton bind. en. $E_{\text {ex }}[\mathrm{meV}]$ & 6 & $1.3-1.4$
\end{tabular}

The function

$$
U\left(r_{c}, r_{v}\right)=-\frac{e^{2}}{\gamma\left|\boldsymbol{r}_{c}-\boldsymbol{r}_{v}\right|}
$$

is the electrostatic interacting potential between the electron and hole, $e$ is the electron's charge, $\gamma=\left(\gamma_{\text {InP }}+\gamma_{\text {InAs }}\right) / 2$, where $\gamma_{\text {InP }}=12.6$ and $\gamma_{\text {InAs }}=15.2$ are the static dielectric constants of $\mathrm{InP}$ and InAs, respectively [25].

Note also that the following notations will be used in all further calculations regarding to the charge carrier's states: $\left|n_{c}\right\rangle$ are the final electron states in the conduction band, $\left|n_{\nu}\right\rangle$ are the states of a hole in the valence band, excluding electron-hole electrostatic interaction, $\left|n_{e}\right\rangle$ is the electron state, which constructs the electrostatic field (see (3)), $\left|n_{\nu}, n_{e}\right\rangle$ are the states of a hole moving in this field.

Since $\mu_{e} / \mu_{h}=1$, the solutions in (2) can be found by the help of the adiabatic approximation [36]. For this purpose, we will present the energy $E^{c, v}=E^{c}+E^{v}$ and wave functions of the pair as follows:

$$
\begin{aligned}
& \Phi\left(r_{c}, r_{v}\right)=\Phi_{c}\left(r_{c}\right) \Phi_{v}\left(r_{v}\right) \\
& \Phi_{i}\left(r_{i}\right)=\chi_{i}\left(r_{i}\right) / r_{i} \quad i=c, v .
\end{aligned}
$$

Now, (2) splits, into the "fast" - electron parts and "slow" - hole parts, respectively

$$
\begin{aligned}
& -\frac{\hbar^{2}}{2 \mu_{e} L^{2}} \frac{\mathrm{d}^{2} \chi_{n_{c}}\left(r_{c}\right)}{\mathrm{d} r_{c}^{2}}=E_{\text {conf }}^{c} \chi_{n_{c}}\left(r_{c}\right), \\
& \chi_{n_{c}}\left(r_{c}=R_{1}\right)=\chi_{n_{c}}\left(r_{c}=R_{2}\right)=0, \\
& -\frac{\hbar^{2}}{2 \mu_{h} L^{2}} \frac{\mathrm{d}^{2} \chi_{n_{v}, n_{e}}\left(r_{v}\right)}{\mathrm{d} r_{v}^{2}}+\bar{U}_{n_{e}}\left(r_{v}\right) \chi_{n_{v}, n_{e}}\left(r_{v}\right)= \\
& E_{n_{v}, n_{e}}^{v} \chi_{n_{v}, n_{e}}\left(r_{v}\right), \\
& \chi_{n_{v}, n_{e}}\left(r_{v}=R_{1}\right)=\chi_{n_{v}, n_{e}}\left(r_{v}=R_{2}\right)=0 .
\end{aligned}
$$

Here, $\bar{U}_{n_{e}}\left(r_{v}\right)$ is the potential of electron-hole interaction averaged over the electronic states of (5) [37] from (3):

$$
\bar{U}_{n_{e}}\left(r_{v}\right)=-\frac{e^{2}}{\gamma} \int \frac{\left|\Phi_{n_{e}}\left(r_{c}\right)\right|^{2}}{\left|\boldsymbol{r}_{c}-\boldsymbol{r}_{v}\right|} \mathrm{d} \boldsymbol{r}_{c} .
$$

Equation (5) is solved elementarily [36] and for the envelope wave functions and energy we get 


$$
\begin{aligned}
& \Phi_{n_{c}}\left(r_{c}\right)=\sqrt{\frac{2}{L}} \frac{\sin \left(\pi n_{c}\left(r_{c}-R_{1}\right) / L\right)}{r_{c}}, \\
& E_{\mathrm{conf}}^{c} \equiv E_{n_{c}}^{c}=\frac{\pi^{2} \hbar^{2} n_{c}^{2}}{2 \mu_{c} L^{2}}, \quad n_{c}=1,2, \ldots
\end{aligned}
$$

In the absence of electron-hole interaction, the envelope wave functions of holes will also have a similar form [35, 36]. Analytical calculations and numerical estimates show that with the considered geometrical dimensions of the structure, the potential $\bar{U}_{n_{e}}\left(r_{v}\right)$ from $(7)$ can be extrapolated by a straight line within the layer (see Appendix A):

$$
\bar{U}_{n_{e}}\left(r_{v}\right) \rightarrow \tilde{U}_{n_{e}}\left(\rho_{v}\right)=-a_{n_{e}}+b_{n_{e}} \rho_{v}, \quad\left(\rho_{v}=\frac{r_{v}}{a_{e x}}\right) .
$$

Solving the corresponding Schrödinger equation (see Appendix B), we obtain the values of the energy of a hole moving in the field of the averaged electron potential (9). Table II shows the values of the hole energies $E_{n_{v}}^{v}$ and $E_{n_{v}, n_{e}}^{v}\left(n_{v}, n_{e}, n_{c}=1,2\right)$ at the different values of the InAs layer thickness without and with taking into account the average potential (A2), (A3).

As can be seen from Table II, the value of the difference $E_{n_{v}=n_{c}}^{v}-E_{n_{v}}^{v}$ increases with the increase of the layer thickness. But, simultaneously with the increase of the layer thickness, this growth slows down with a tendency toward a certain limit. The first circumstance is due to the fact that with the increase of the layer thickness the role of size quantization decreases, but, at the same time, the role of the electrostatic interaction between the electron and the hole increases. The second circumstance is due to the fact that when the layer thickness increases, and it becomes approximately equal to the radius of the bulk exciton, potential (7) already less accurately describes the state of the pair, and the electronhole interaction must now be taken into account within a more accurate model, for example the Wannier-Mott exciton model. When the layer thickness is less than the Bohr radius of the exciton, size quantization is dominant,

\section{TABLE II}

The values of the hole energy $E_{n_{v}}^{v}$ and $E_{n_{v}, n_{e}}^{v}$, $\left(n_{v}, n_{e}=1,2\right)$ in the InAs layer without and with taking into account the electron-hole interaction for different values of the layer thickness $L$ (energy is measured from zero level)

\begin{tabular}{c|c|c|c|c}
\hline \hline \multicolumn{1}{c|}{$L[\mathrm{~nm}]$} & 20 & 25 & 30 & 35 \\
\hline$E_{1}^{v}[\mathrm{meV}]$ & 2.2867 & 1.4635 & 1.0163 & 0.7467 \\
$E_{2}^{v}[\mathrm{meV}]$ & 9.1468 & 5.8540 & 4.0653 & 2.9867 \\
$E_{1,1}^{v}[\mathrm{meV}]$ & 2.9702 & 2.1814 & 1.7493 & 1.5417 \\
$E_{2,1}^{v}[\mathrm{meV}]$ & 9.8134 & 6.5992 & 5.2418 & 3.7120 \\
$E_{1,2}^{v}[\mathrm{meV}]$ & 3.0406 & 2.3008 & 1.8739 & 1.5667 \\
$E_{2,2}^{v}[\mathrm{meV}]$ & 9.8665 & 6.6596 & 4.9173 & 3.8759 \\
$E_{1,1}^{v}-E_{1}^{v}$ & 0.6835 & 0.7179 & 0.7830 & 0.7950 \\
$E_{2,2}^{v}-E_{2}^{v}$ & 0.7197 & 0.8056 & 0.8520 & 0.8892
\end{tabular}

and the electrostatic interaction is taken into account as a correction. When the layer thickness becomes of the order of the Bohr radius of the exciton, the electrostatic interaction becomes a dominant. As a result, the approximation proposed in this work becomes already inaccurate for such values of the layer thickness.

\section{Discussion of results}

As the illustration of the $e-h$ electrostatic interaction effect on the properties of the nanocomposite under consideration, we discuss interband optical transitions and luminescence in this structure.

\subsection{Interband optical transitions}

Let us consider the interband optical dipole transitions between the states of valence $|v\rangle$ and conductive $|c\rangle$ bands and examine the influence of potential (3) on these transitions. For the perturbation $A$, related to the light wave, we will have in dipole approximation:

$$
A=\frac{|q| A_{0}}{m_{0} c}\left(\boldsymbol{r}_{e} \cdot \boldsymbol{r}_{p}\right) .
$$

Here $A_{0}$ is the amplitude of vector-potential, $\boldsymbol{p}$ is a $3 \mathrm{D}$ operator of momentum, $m_{0}$ is the free electron mass and $c$ is wave's velocity in free space. For the matrix element $M_{c, v}$ of interband $\left|n_{v}, l_{v}=0, m_{v}=0\right\rangle \rightarrow\left|n_{c}, l_{c}=0, m_{c}=0\right\rangle$ transitions without considering electron-hole interaction, one can write $[2,3]$ :

$$
M_{c, v}=A_{c, v}\left\langle\Phi_{n_{c}}(r) \mid \Phi_{n_{v}(r)}\right\rangle \delta_{n_{c}, n_{v}}
$$

where $A_{c, v}$ is the matrix element of operator (10), built on the Bloch amplitudes of $v$ - and $c$-bands and envelope radial functions $\Phi_{n}(r)$ are taken from (8). Non-diagonal interband optical transitions $\left(n_{c} \neq n_{v}\right)$ in the absence of $e-h$ interaction are prohibited. The threshold energy $\hbar \Omega_{c, v}^{(0)}$ of the interband absorption in this case is determined by the size quantization energies of electron and hole

$$
\hbar \Omega_{n_{c}, n_{v}}^{(0)}=E_{g}+E_{n_{c}}+E_{n_{v}} .
$$

Calculations using wave functions from (8) and (B.2) show that the potential (3) removes the selection rules (11). Accordingly, in the presence of $e^{-} h$ electrostatic interaction, along with diagonal transitions in the considered structure, non-diagonal interband transitions are also possible. The intensity of diagonal transitions when taking into account the $e-h$ interaction in relative units is equal to one. The intensity of non-diagonal transitions turns out to be less than one. Here, obviously, is a direct analogy with interband transitions in similar nanostructures in the presence of a uniform electrostatic field (see for example $[3,37]$ ). This is due to the framework of our approximation: indeed, the potential (A.2), (A.3) is quasi-homogeneous in its behavior.

Table III shows the oscillator strengths $K_{c, v} \equiv K_{n_{c}, n_{v}, n_{e}}$ (in relative units) for non-diagonal interband transitions in the presence of $e-h$ interaction for different values of film thickness. As we have noticed, 
TABLE III

Values of oscillator strengths $K_{c, v} \equiv K_{n_{c}, n_{v}, n_{e}}$ (in relative units) of non-diagonal interband transitions in the $\mathrm{InP} / \mathrm{InAs} / \mathrm{InP}$ heterostructure in the presence of $e-h$ interaction for different values of the film thickness $L$.

\begin{tabular}{l|c|c|c|c}
\hline \hline \multicolumn{1}{c|}{$L[\mathrm{~nm}]$} & 20 & 25 & 30 & 35 \\
\hline $\begin{array}{l}\left|n_{v}=1, n_{c}=1\right\rangle \rightarrow\left|n_{c}=2\right\rangle \\
K_{2 ; 1,1}\end{array}$ & 0.0361 & 0.0597 & 0.0148 & 0.1214 \\
\hline $\begin{array}{l}\left|n_{v}=2, n_{c}=1\right\rangle \rightarrow\left|n_{c}=1\right\rangle \\
K_{1 ; 2,1}\end{array}$ & 0.0337 & 0.0601 & 0.0656 & 0.1114 \\
\hline $\begin{array}{l}\left|n_{v}=1, n_{c}=2\right\rangle \rightarrow\left|n_{c}=2\right\rangle \\
K_{2 ; 1,2}\end{array}$ & 0.0486 & 0.1013 & 0.0670 & 0.1579 \\
\hline $\begin{array}{l}\left|n_{v}=2, n_{c}=2\right\rangle \rightarrow\left|n_{c}=1\right\rangle \\
K_{1 ; 2,2}\end{array}$ & 0.0367 & 0.0651 & 0.0070 & 0.1383
\end{tabular}

TABLE IV

The values of the threshold energies of the interband absorption in the $\mathrm{InP} / \mathrm{InAs} / \mathrm{InP}$ heterocomposite without taking into account $\left(\hbar \Omega_{n_{c}, n_{v}}^{(0)}\right)$ and taking into account the $e$ - $h$ interaction $\left(\hbar \Omega_{n_{c} ; n_{v}, n_{c}}^{e-h}\right)$ for the different values of the layer thickness $L$ [nm].

\begin{tabular}{l|c|c|c|c}
\hline \hline \multicolumn{1}{c|}{$L$} & 20 & 25 & 30 & 35 \\
\hline$\hbar \Omega_{1,1}^{(0)}[\mathrm{meV}]$ & 397.05 & 381.55 & 373.13 & 368.06 \\
$\hbar \Omega_{2,2}^{(0)}[\mathrm{meV}]$ & 526.20 & 464.21 & 430.53 & 410.23 \\
$\hbar \Omega_{1 ; 1,1}^{e-h}[\mathrm{meV}]$ & 391.75 & 376.66 & 368.59 & 363.79 \\
$\hbar \Omega_{2 ; 1,1}^{e-h}[\mathrm{meV}]$ & 514.03 & 454.93 & 423.12 & 403.73 \\
$\hbar \Omega_{1 ; 1,2}^{e-h}[\mathrm{meV}]$ & 391.74 & 377.05 & 368.59 & 363.75 \\
$\hbar \Omega_{2 ; 1,2}^{e-h}[\mathrm{meV}]$ & 514.03 & 455.31 & 422.94 & 403.68 \\
$\hbar \Omega_{2 ; 2,1}^{e-h}[\mathrm{meV}]$ & 520.80 & 459.35 & 426.43 & 405.96 \\
$\hbar \Omega_{2 ; 2,2}^{e-h}[\mathrm{meV}]$ & 520.86 & 459.31 & 425.96 & 405.99 \\
$\hbar \Omega_{1 ; 2,2}^{e-h}[\mathrm{meV}]$ & 398.57 & 381.04 & 371.59 & 366.06 \\
$\hbar \Omega_{1 ; 2,1}^{e-h}[\mathrm{meV}]$ & 398.51 & 381.08 & 372.08 & 366.03 \\
$\hbar \Omega_{1 ; 1,1}^{e-h}-\hbar \Omega_{1,1}^{(0)}$ & 5.3 & 4.9 & 4.54 & 4.27 \\
$\hbar \Omega_{2 ; 2,2}^{e-h}-\hbar \Omega_{2,2}^{(0)}$ & 5.34 & 4.9 & 4.57 & 4.24
\end{tabular}

the intensity of non-diagonal transitions on one-two order is less than intensity of diagonal transitions.

Taking into account $e-h$ interaction also leads to the decrease of the interband absorption threshold energy values. Using data from Tables I and II, and (A.1), and expressions (8), (9), (12), the values of threshold energies are calculated without taking into account $\left(\hbar \Omega_{n_{c}, n_{v}}^{(0)}\right)$ and taking into account $\left(\hbar \Omega_{n_{c}, n_{v}, n_{c}}^{e-h}\right)$ the $e-h$ interaction. The corresponding values of these frequencies are given in Table IV.

Table IV clearly shows that taking into account the $e-h$ interaction leads to a decrease of the threshold energies compared with the approximation without considering the interaction. At the same time, it should be noted that the following transitions:

$\left(\left|n_{\nu}=1, n_{e}=1\right\rangle \rightarrow\left|n_{c}=1\right\rangle,\left|n_{\nu}=1, n_{e}=2\right\rangle \rightarrow\left|n_{c}=1\right\rangle\right)$,

$\left(\left|n_{\nu}=2, n_{e}=1\right\rangle \rightarrow\left|n_{c}=2\right\rangle,\left|n_{\nu}=2, n_{e}=2\right\rangle \rightarrow\left|n_{c}=2\right\rangle\right)$,

$\left(\left|n_{\nu}=2, n_{e}=1\right\rangle \rightarrow\left|n_{c}=1\right\rangle,\left|n_{\nu}=2, n_{e}=2\right\rangle \rightarrow\left|n_{c}=1\right\rangle\right)$ in pairs have the same threshold energy. This is explained by the fact that the averaged electrostatic field (7) created by an electron is determined only by the localization region of the electron, but not by its velocity. Mathematically this is manifested by the fact that the average value of the electron probability density $\left|\chi_{n_{c}}\left(r_{c}\right)\right|^{2}$ throughout the entire interval $r_{c} \in\left[R_{1}, R_{2}\right]$ has the same value, regardless of the state number. In addition, the difference between the threshold energies of the diagonal transitions without and with taking into account the $e-h$ interaction has the same value: $\hbar \Omega_{1,1,1}^{e-h}-\hbar \Omega_{1,1}^{(0)}=\hbar \Omega_{2,2,2}^{e-h}-\hbar \Omega_{2,2}^{(0)}$. This is also a consequence of the factor that the field contribution of the electronic states $\left|n_{e}=1\right\rangle$ and $\left|n_{e}=2\right\rangle$ to the energy of motion of a hole has the same value for different values of quantum number $n_{e}$.

Now let us consider the interband optical absorption in the real ensemble of core/shell/shell nanostructures with the dispersion of their sizes in the strong size quantization regime. Note that this dispersion will lead to broadenings of spectral lines. According to [37] we will have the following expression for light absorption coefficient for the strong quantization regime:

$$
\alpha(\omega)=\alpha_{0} \sum_{\nu_{e}, \nu_{h}} J^{2} \delta\left(\hbar \omega_{e, h}-E_{g}^{L}-E_{e}-E_{h}\right),
$$

where $E_{g}$ is the band gap of the semiconductor, $\alpha_{0}$ is a quantity proportional to the square of the dipole moment's matrix element taken over the Bloch functions. The broadenings of spectral lines will be taken into consideration replacing the delta function in (1) by the Lorentz contour

$$
\begin{gathered}
\delta\left(\hbar \omega_{e, h}-E_{g}^{L}-E_{e}-E_{h}\right) \longrightarrow \\
\frac{\Gamma}{\left(\hbar \omega_{e, h}-E_{g}^{L}-E_{e}-E_{h}\right)^{2}+\Gamma^{2}},
\end{gathered}
$$

where $\Gamma$ is the width of Lorentzian parameter. For calculations we take $\Gamma=5 \mathrm{meV}$, according to [38].

The next step of the investigation of the $\mathrm{InP} / \mathrm{InSb} / \mathrm{InP}$ core/shell/shell structure's optical properties is the calculation of the photoluminescence (PL) spectra. The PL spectra can be calculated using the Roosbroeck-Shockley relation $[39,40]$ :

$$
R(\hbar \omega)=R_{0} \hbar \omega \alpha(\hbar \omega) \frac{f_{c}\left(1-f_{v}\right)}{f_{v}-f_{c}},
$$

where $R_{0}$ is proportional to the square of the matrix element of the dipole moment taken over the Bloch functions, $f_{c}$ and $1-f_{v}$ are probabilities of the conduction band states being occupied and the valence band states being empty, respectively. For the high temperatures the term $\frac{f_{c}\left(1-f_{v}\right)}{f_{v}-f_{c}}$ in the Roosbroeck-Shockley relation transforms into the Boltzmann-like form.

Note that the PL curves have been calculated for the room temperature. The separate peaks corresponding to the transitions described in the absorption part can be seen in the PL spectra. As we mentioned above, for the high temperatures the term $\frac{f_{c}\left(1-f_{v}\right)}{f_{v}-f_{c}}$ in the RoosbroeckShockley relation transforms into the Boltzmann-like 

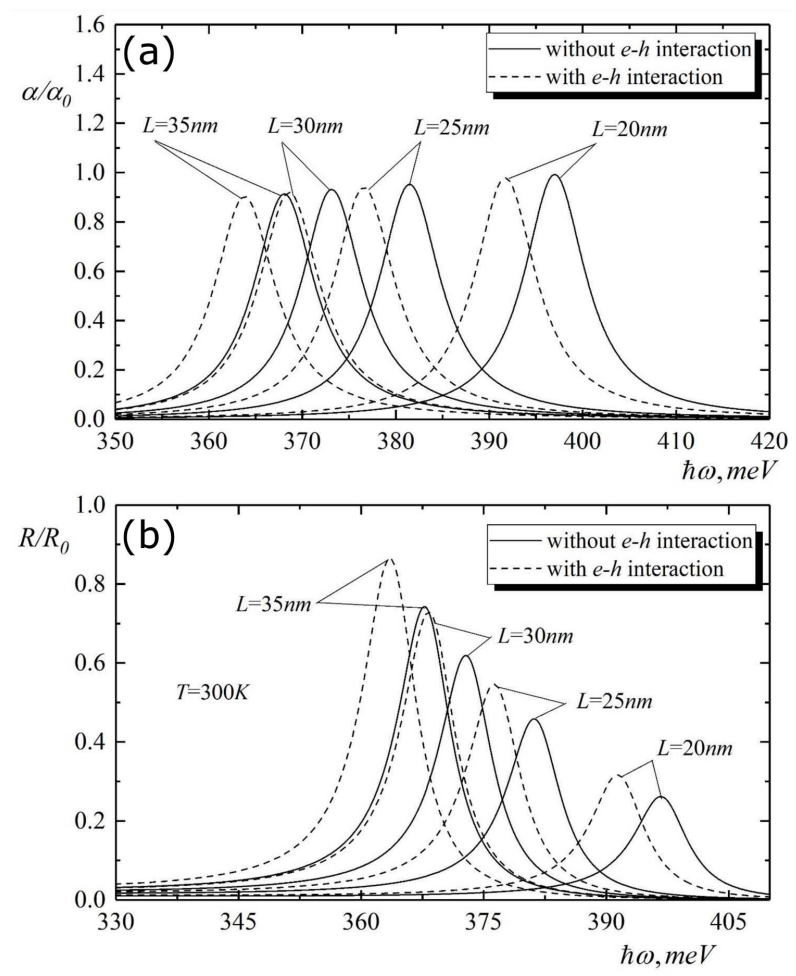

Fig. 1. The dependence of the absorption coefficient (a) and photoluminescence spectra (b) on the incident light frequency for the diagonal transitions $\left|n_{\nu}=1, n_{e}=1\right\rangle \rightarrow\left|n_{c}=1\right\rangle$.

form and this term vanishes the peaks in the high-energy region. That is why the low energy peaks can be observed more clearly in the PL spectra than the highenergy peaks $[41,42]$. It is also obvious that the peaks corresponding to the diagonal transitions have higher intensity than the peaks corresponding to the non-diagonal transitions.

The dependences of the absorption coefficient for the diagonal transitions $\left|n_{\nu}=1, n_{e}=1\right\rangle \rightarrow\left|n_{c}=1\right\rangle$ are presented in Fig. 1. As we can see from Fig. 1, with the increase of thickness $L$ the peak positions have red shift. In the same time the small increase of the peak intensities has been considered with the decrease of thickness of layer. For the fixed value of the thickness taking into account the $e-h$ interaction leads to the red shift of peak positions. The same behavior for diagonal transitions $\left|n_{\nu}=1, n_{e}=1\right\rangle \rightarrow\left|n_{c}=1\right\rangle$ can be seen also for the PL spectra, which is presented in Fig. 1b. With the increase of thickness $L$ the intensities of PL spectra increase.

As we noted above, accounting of $e-h$ interaction the non-diagonal transitions are possible in the system. The dependences of the absorption coefficient for the nondiagonal transitions $\left|n_{\nu}=2, n_{e}=1\right\rangle \rightarrow\left|n_{c}=1\right\rangle$ are presented in Fig. 2. With the increase of thickness $L$ the peak positions for both absorption and PL spectra have red shift. The values of intensities for non-diagonal transitions, which are one-two order less than intensities of diagonal transitions, are presented in Table III.
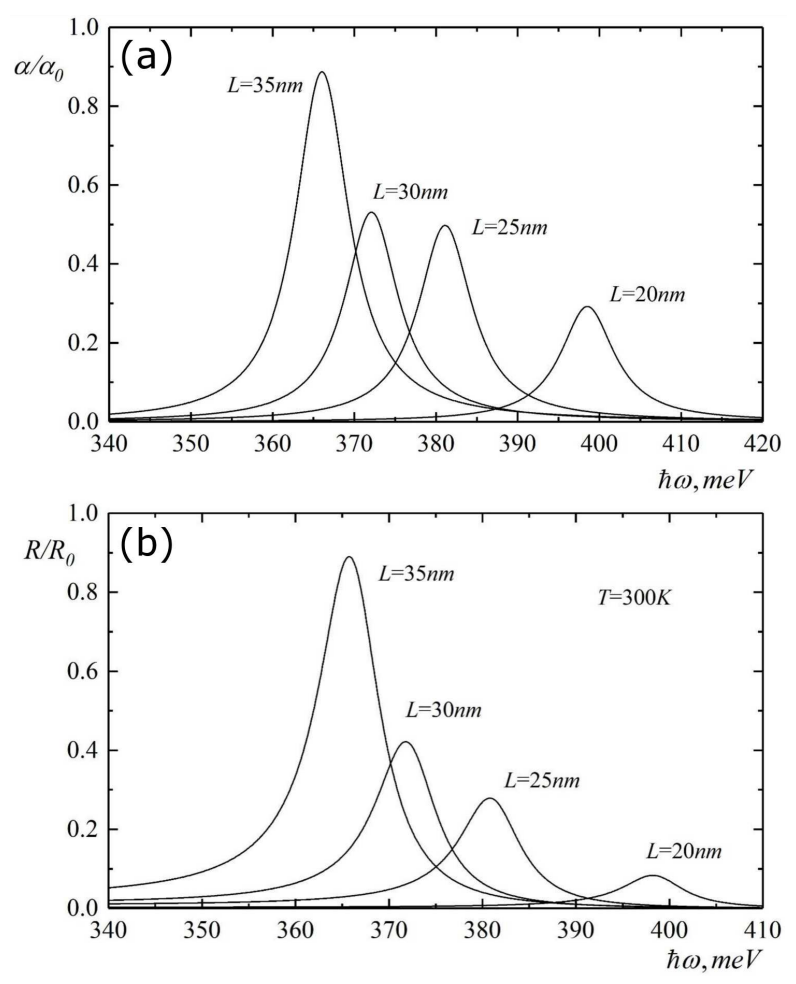

Fig. 2. As in Fig. 1, but for the non-diagonal transitions.

\section{Conclusions}

For the considered geometric dimensions of the system consideration of the electrostatic electron-hole interaction is physically necessary. The presence of electrostatic $e-h$ interaction increases the absolute value of the hole energy in the quantum well. Hence, the total energy of the pair also increases. Under the influence of the electrostatic potential of the electron, the hole is pressed to the region of the inner radius of the layer. Electrostatic $e-h$ interaction removes the selection rules for the radial number for interband optical transitions. The electrostatic $e-h$ interaction leads to the shift of the threshold energy of interband transitions. The magnitude of the $e-h$ interaction potential can be adjusted by varying the thickness of the quantizing layer of the core/shell/shell structure, which can be used to control the band frequencies of the absorbed radiation.

\section{Appendix A: The calculation of the potential $\bar{U}_{n_{c}}\left(r_{v}\right)$ from (7).}

To calculate the integral, we proceed to the triangular coordinates, where

$$
\left|\boldsymbol{r}_{c}-\boldsymbol{r}_{v}\right|=\sqrt{r_{c}^{2}+r_{v}^{2}-2 r_{c} r_{v} \cos (\alpha)} .
$$

Here, $\alpha$ is the angle between vectors $\boldsymbol{r}_{c}$ and $\boldsymbol{r}_{v}$. Substituting (A.1) into (7) and breaking the entire integration interval by the radial variable $r_{c} \in\left[R_{1}, R_{2}\right]$ into two intervals $r_{c} \in\left[R_{1}, r_{v}\right]$ and $r_{c} \in\left[r_{v}, R_{2}\right]$ we obtain the following expression for the potential: 


$$
\bar{U}_{n_{e}}\left(r_{v}\right)=-\frac{2}{L} \frac{e^{2}}{\gamma}\left\{\begin{array}{l}
\left(1-\frac{R_{1}}{r_{v}}\right)+\ln \left(\frac{R_{2}}{r_{v}}\right)-\frac{L}{2 \pi n_{e} r_{v}} \sin \left(\frac{2 \pi n_{e}\left(r_{v}-R_{1}\right)}{L}\right) \\
-\cos \left(\frac{2 \pi n_{e} R_{1}}{L}\right)\left[\cos \operatorname{int}\left(\frac{2 \pi n_{e} R_{2}}{L}\right)-\operatorname{cosint}\left(\frac{2 \pi n_{e} r_{v}}{L}\right)\right] \\
-\sin \left(\frac{2 \pi n_{e} R_{1}}{L}\right)\left[\sin \operatorname{int}\left(\frac{2 \pi n_{e} R_{2}}{L}\right)-\sin \operatorname{int}\left(\frac{2 \pi n_{e} r_{v}}{L}\right)\right]
\end{array}\right\}
$$

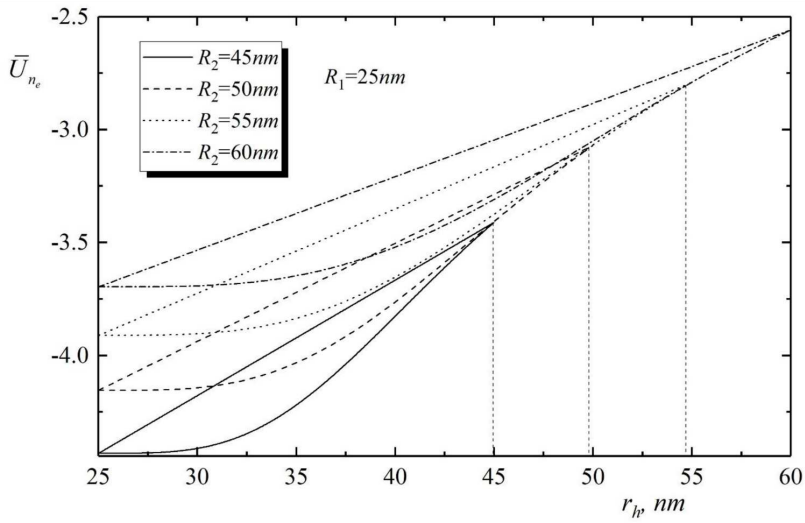

Fig. A1. Plots of functions $\bar{U}_{n_{e}=1}\left(r_{v}\right)$ in dimensionless units at a fixed value of $R_{1}=25 \mathrm{~nm}$ and $R_{2}=45,50$, $55,60 \mathrm{~nm}$.

TABLE A1

Values of parameters $a_{n_{e}}(L)$ and $b_{n_{e}}(L)$ when thickness of the layer $L=20,25,30,35 \mathrm{~nm}\left(n_{e}=1,2\right)$.

\begin{tabular}{c|c|c|c|c}
\hline \hline$L$ & 20 & 25 & 30 & 35 \\
\hline$a_{1}(L)$ & -5.7111 & -5.2391 & -4.8443 & -4.5086 \\
$b_{1}(L)$ & 1.7893 & 1.5187 & 1.3069 & 1.1379 \\
$a_{2}(L)$ & -5.8386 & -5.3851 & -5.0056 & -4.6824 \\
$b_{2}(L)$ & 1.8884 & 1.6209 & 1.4096 & 1.2392
\end{tabular}

For convenience, in all further intermediate calculations, the value of $a_{\mathrm{ex}}=35 \mathrm{~nm}$ is used as the unit of length, and the value of $E_{\text {et }}=\hbar^{2} / 2 \mu_{c} a_{\mathrm{ex}}^{2}=1.3501 \mathrm{meV}$ is used as the unit of energy. Analysis of the function $\bar{U}_{n_{e}}\left(r_{v}\right)$ behavior from (A.2) shows that in the range of values of a variable $r_{v} \in\left[R_{1}, R_{2}\right]$, this function increases monotonically, taking its smallest and largest values at the points $r_{v}=R_{1}$ and $r_{v}=R_{2}$, respectively.

Figure A1 shows the graphs of the function $\bar{U}_{n_{e}}\left(r_{v}\right)$ in reduced units in the range of values $r_{v} \in\left[R_{1}, R_{2}\right]$ at $R_{1}=25 \mathrm{~nm}$ when $R_{2}=45,50,55,60 \mathrm{~nm}$.

It is not difficult to conclude from this figure that the extrapolation of graphs of functions $\bar{U}_{n_{c}}\left(r_{v}\right)$ in the interval $r_{v} \in\left[R_{1}, R_{2}\right]$ by the corresponding straight line. Indeed, such extrapolation practically does not change the physical essence of the influence of the quantum well bottom profile on the formation of the energy spectrum of holes. The largest deviation between the values of the functions from (A.2) and the straight line

$$
\tilde{U}_{n_{e}}\left(\rho_{v}\right)=-a_{n_{e}}(L)+b_{n_{e}}(L) \rho_{v}, \quad \rho_{v}=\frac{r_{v}}{a_{\mathrm{ex}}}
$$

is less than $7 \%$. The values of the parameters $a_{n_{c}}(L)$ and $b_{n_{c}}(L)$ depend on the layer thickness and are determined numerically. Table A1 shows the values of the parameters $a_{n_{e}}(L)$ and $b_{n_{2}}(L)$ for various values of the layer thickness $L$.

\section{Appendix B: The states of a hole in an InAs} layer in the presence of potential (A.2) and (A.3)

In this case, the Schrödinger radial Eq. (6) with potential (A.2) and (A.3) in the effective mass approximation is reduced to the following equation:

$$
\begin{aligned}
& \chi(\xi)-\xi \chi(\xi)=0 \quad \xi=\left(r_{v}-\frac{E}{F}\right)\left(\frac{2 \mu_{h} F}{\hbar^{2}}\right)^{1 / 3}, \\
& \chi\left(\xi_{1}\right)=\chi\left(\xi_{2}\right)=0 \quad \xi_{1,2}=\left(R_{1,2}-\frac{E}{F}\right)\left(\frac{2 \mu_{h} F}{\hbar^{2}}\right)^{1 / 3}, \\
& F=\frac{b, E_{\mathrm{et}}}{a_{\mathrm{ex}}} .
\end{aligned}
$$

Here, $E=E_{n_{v}, n_{e}}^{v}+a_{n_{e}} E_{\text {et }}$, and $E_{n_{v}, n_{e}}^{v}$ is the required energy of a hole moving in the field of the electron potential (A.2) and (A.3). The solutions of (B.1) are given by the following linear combination:

$$
\chi(\xi)=C_{1} \operatorname{Ai}(\xi)+C_{2} \operatorname{Bi}(\xi) .
$$

Here $\operatorname{Ai}(\xi), \operatorname{Bi}(\xi)$ are the Airy functions of the first and second kind, respectively, and $C_{1,2}$ are the normalizing constants. Figure B1 shows the hole probability density distribution with and without taking into account $e-h$ interaction. As we can see from the figure, if $e-h$ interaction is taken into account, then the probability density distribution peak for hole shifts to the inner radius of the core/shell/shell structure.

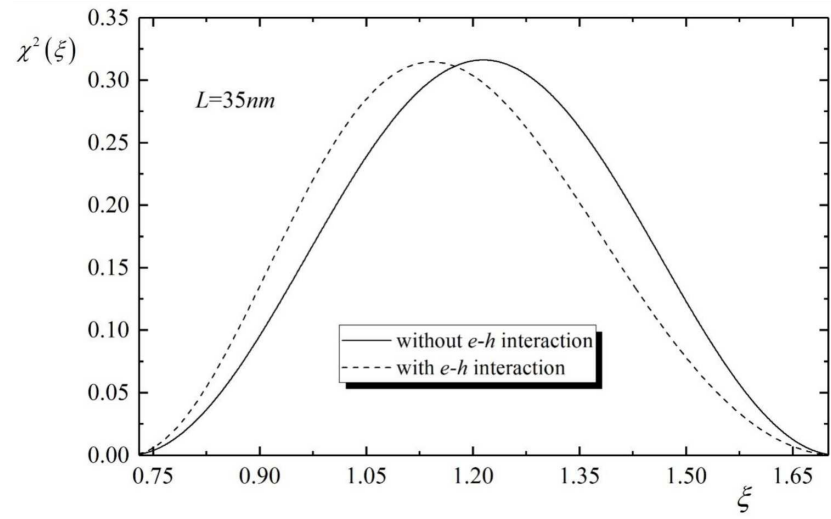

Fig. B1. The hole probability density distribution with and without taking into account $e^{-} h$ interaction at $L=35 \mathrm{~nm}$. 
Using (A.1), (B.1) and (B.2) one can write the following equation for the definition of hole energy spectrum:

$$
\begin{gathered}
\frac{\operatorname{Ai}\left[\left(\frac{\mu_{h}}{\mu_{e}}\right)^{1 / 3}\left(\rho_{1} b_{n_{e}}^{1 / 3}-\varepsilon_{n_{v}, n_{e}} b_{n_{e}}^{-2 / 3}\right)\right]}{\operatorname{Ai}\left[\left(\frac{\mu_{h}}{\mu_{e}}\right)^{1 / 3}\left(\rho_{2} b_{n_{e}}^{1 / 3}-\varepsilon_{n_{v}, n_{e}} b_{n_{e}}^{-2 / 3}\right)\right]}= \\
\frac{\operatorname{Bi}\left[\left(\frac{\mu_{h}}{\mu_{e}}\right)^{1 / 3}\left(\rho_{1} b_{n_{e}}^{1 / 3}-\varepsilon_{n_{v}, n_{e}} b_{n_{e}}^{-2 / 3}\right)\right]}{\operatorname{Bi}\left[\left(\frac{\mu_{h}}{\mu_{e}}\right)^{1 / 3}\left(\rho_{2} b_{n_{e}}^{1 / 3}-\varepsilon_{n_{v}, n_{e}} b_{n_{e}}^{-2 / 3}\right)\right]} \\
\rho_{1,2}=\frac{R_{1,2}}{a_{\mathrm{ex}}}, \quad \varepsilon_{n_{v}, n_{e}}=\frac{E_{n_{v}, n_{e}}^{v}}{E_{\mathrm{et}}}+\left|a_{n_{e}}\right| .
\end{gathered}
$$

\section{Acknowledgments}

This work was supported by the RA MES State Committee of Science, in the frames of the research project No. $18 \mathrm{~T}-1 \mathrm{C} 062$

\section{References}

[1] H.E. Porţeanu, E. Lifshitz, M. Pflughoefft, A. Eychmüller, H. Weller, Phys. Status Solidi B 226, 219 (2001).

[2] V.A. Harutyunian, Thin Solid Films 446, 258 (2004).

[3] V.A. Harutyunyan, Physica E 39, 37 (2007).

[4] P. Reiss, M. Protière, L. Li, Small 5, 154 (2009).

[5] D. Andrews, G. Scholes, G. Wiederrecht, Comprehensive Nanoscience and Technology, Academic Press, 2010.

[6] R.G. Chaudhuri, S. Paria, Chem. Rev. 112, 2373 (2012).

[7] A. Ibral, A. Zouitine, E.-M. Assaid, H.-E. Achouby, E. Feddi, F. Dujardin, Physica B Condens. Matter 458, 73 (2014).

[8] D. Ramaccia, S. Arcieri, A. Toscano, F. Bilotti, in: Proc. 10th Int. Congress on Advanced Electromagnetic Materials in Microwaves and Optics, Chania (Greece), 2016, p. 298.

[9] V.A. Harutyunyan, D.A. Baghdasaryan, D.B. Hayrapetyan, Physica B 510, 33 (2017).

[10] V.A. Harutyunyan, D.B. Hayrapetyan, E.M. Kazaryan, Adv. Mater. Sci. Eng. 2, 1 (2018).

[11] K.J. Bacmann, Ann. Rev. Mater. Sci. 11, 441 (1981).

[12] T. Suemitsu, IEICE Electron. Express 12, 1 (2015).

[13] K. Ikossi, InAs Device Process Development and Characterization, Naval Research Laboratory, Washington 2003, p. 43.

[14] A. Hayfaa, Ph.D. Thesis, Lancaster University, 2018, p. 146.

[15] N.A.J.M. Kleemans, J. van Bree, M. Bozkurt et al., Phys. Rev. B 79, 045311 (2009).

[16] M. Helmi, H. Alouane, R. Anufriev, N. Chauvin, C. Bru-Chevallier, et al., Nanotechnology 22, 405702 (2011).

[17] J. van Bree, A.Yu. Silov, P.M. Koenraad, M.E. Flatté, C.E. Pryor, Phys. Rev. B 85, 165323 (2012).
[18] M. Zahed, M. Khan, T. Kheeng, B.S. Ooi, Progr. Quant. Electron. 38, 237 (2014).

[19] X. Yan, S. Fan, X. Zhang, X. Ren, Nanoscale Res. Lett. 10, 389 (2015).

[20] M. Fu, Electrical Properties of Indium Arsenide Nanowires and Their Field-Effect Transistors, Springer, Singapore 2018, p. 101.

[21] P.J. Delfyett, A. Ardey, S.P. Bhooplapur, E. Sarailou, IEEE J. Select. Top. Quant. Electron. 24, 1 (2018).

[22] X. Ji, C. Xiren, X. Yang, T. Yang, Nanoscale Res. Lett. 13, 269 (2018).

[23] Y. Masumoto, K. Goto, S. Yoshida, Y. Sakuma, P. Mohan, J. Motohisa, T. Fukui, Phys. Rev. B 82, 075313 (2010).

[24] M. Royo, J. Planelles, Phys. Rev. B 75, 033302 (2007).

[25] E. Assaid, E. Feddi, J. El Khamkhami, F. Dujardin, J. Phys. Condens. Matter 15, 175 (2003).

[26] V.I. Klimov, Semiconductor and Metal Nanocrystals: Synthesis and Electronic and Optical Properties, CRC Press, 2003, p. 500.

[27] P. Bhardwaj, N. Das, Int. J. Eng. Sci. Res. Technol. 5, 289 (2016)

[28] Ioffe Institute, InP — Indium Phosphide: Basic Parameters at $300 \mathrm{~K}$.

[29] Ioffe Institute, InP - Indium Phosphide: Band Structure and Carrier Concentration.

[30] W.E. Buhro, V.L. Colvin, Nature Mater. 2, 138 (2003).

[31] Y. Wang, X. Yang, T.C. He, Y. Gao, H.V. Demir, X.W. Sun, H.D. Sun, Appl. Phys. Lett. 102, 021917 (2013).

[32] Ioffe Institute, InAs — Indium Arsenide: Basic Parameters at $300 \mathrm{~K}$.

[33] Ioffe Institute, InAs - Indium Arsenide: Band Structure and Carrier Concentration.

[34] M.H. Sun, E.S.P. Leong, A.H. Chin, C.Z. Ning, G.E. Cirlin, Yu.B. Samsonenko, V.G. Dubrovskii, L. Chuang, C. Chang-Hasnain, Nanotechnology 21, 335705 (2010).

[35] V.A. Harutyunyan, M.A. Mkrtchyan, E.M. Kazaryan, D.B. Hayrapetyan, J. Contemp. Phys. 54, 33 (2019).

[36] V. Harutyunyan, Effect of Static Electric Fields on the Electronic and Optical Properties of Layered Semiconductor Nanostructures, Bentham Sci. Publ., 2015, Ch. 1, p. 242.

[37] Al.L. Efros, A.L. Efros, Semiconductors 16, 772 (1982).

[38] A.B. Djurišić, E.H. Li, D. Rakić, M.L. Majewski, Appl. Phys. A 70, 29 (2000).

[39] W. Van Roosbroeck, W. Shockley, Phys. Rev. 94, 1558 (1954).

[40] R. Bhattacharya, B. Pal, B. Bansal, Appl. Phys. Lett. 100, 222103 (2012).

[41] V.A. Harutyunyan, D.B. Hayrapetyan, E.M. Kazaryan, J. Contemp. Phys. 53, 48 (2018).

[42] D.B. Hayrapetyan, D.A. Baghdasaryan, E.M. Kazaryan, S.I. Pokutnyi, H.A. Sarkisyan, Chem. Phys. 506, 26 (2018). 